\title{
RESULTS OF THE CRYOGENIC TESTS OF THE SUPERCONDUCTING MAGNETS FORMING THE BARREL TOROID OF THE ATLAS EXPERIMENT
}

\author{
K. Barth ${ }^{1}$, N. Delruelle ${ }^{1}$, A. Dudarev ${ }^{1}$, S. Junker ${ }^{1}$, R. Pengo ${ }^{1}$, O. Pirotte ${ }^{1}$, and C. Berriaud ${ }^{2}$
}

The Barrel Toroid magnet of the ATLAS experiment will be built from eight $25 \mathrm{~m} \times 5 \mathrm{~m}$ racetrack shaped superconducting coils that are symmetrically placed around the central axis of the detector. Prior to their final assembly in the underground cavern of the LHC, these magnets are individually tested at ground level in order to verify the expected overall performances. A dedicated facility has been commissioned and the testing of the coils, at their nominal electrical and thermal operating conditions, has been carried out. The paper presents the results obtained during the cool-down phase from ambient temperature, the steady-state operation at $4.5 \mathrm{~K}$, the $20 \mathrm{kA}$ current ramping up/down and the thermal recovery after a fast energy dump of up to $138 \mathrm{MJ}$ stored energy. Included are the measurements of the various thermal loads in both static and dynamic conditions.

1 CERN, Accelerator Technology department and Physics Departement, Geneva, Switzerland

2 CEA-SACLAY, France 


\title{
RESULTS OF THE CRYOGENIC TESTS OF THE SUPERCONDUCTING MAGNETS FORMING THE BARREL TOROID OF THE ATLAS EXPERIMENT
}

\author{
K. Barth ${ }^{1}$, N. Delruelle ${ }^{1}$, A. Dudarev ${ }^{1}$, S. Junker ${ }^{1}$, R. Pengo ${ }^{1}$, O. Pirotte ${ }^{1}$, \\ and C. Berriaud ${ }^{2}$ \\ ${ }^{1}$ Accelerator Technology department-CERN, Geneva, Switzerland \\ ${ }^{2}$ CEA-SACLAY, France
}

\begin{abstract}
The Barrel Toroid magnet of the ATLAS experiment will be built from eight $25 \mathrm{~m} \times 5$ $m$ racetrack shaped superconducting coils that are symmetrically placed around the central axis of the detector. Prior to their final assembly in the underground cavern of the LHC, these magnets are individually tested at ground level in order to verify the expected overall performances. A dedicated facility has been commissioned and the testing of the coils, at their nominal electrical and thermal operating conditions, has been carried out. The paper presents the results obtained during the cool-down phase from ambient temperature, the steady-state operation at $4.5 \mathrm{~K}$, the $20 \mathrm{kA}$ current ramping up/down and the thermal recovery after a fast energy dump of up to $138 \mathrm{MJ}$ stored energy. Included are the measurements of the various thermal loads in both static and dynamic conditions.
\end{abstract}

KEYWORDS: Cryogenics, Superconducting Magnets, Centrifugal Pumps, Heat Loads. PACS: 07.20.Mc, 81.70.Ex, 47.85.g, 44.40.+a, 05.70.C, 65.40.Gr, 67.20.+k, 84.71.Ba, 84.71.

\section{INTRODUCTION}

The ATLAS superconducting magnet system is composed of a Barrel Toroid (BT), two End Cap Toroids (ECT's) and a central solenoid. Eight large (25 m x 5 m) superconducting magnets of the race-track type form the BT: each has been tested at ground level in a facility constructed for this purpose. The same facility has been used to carry out the test of the prototype magnet B0.

The aim of the tests was to validate the electromagnetic and cryogenic performance of each magnet. In this paper the latter will be described, i.e. the heat loads of the cold masses, both static and dynamic, and of the thermal shields. 


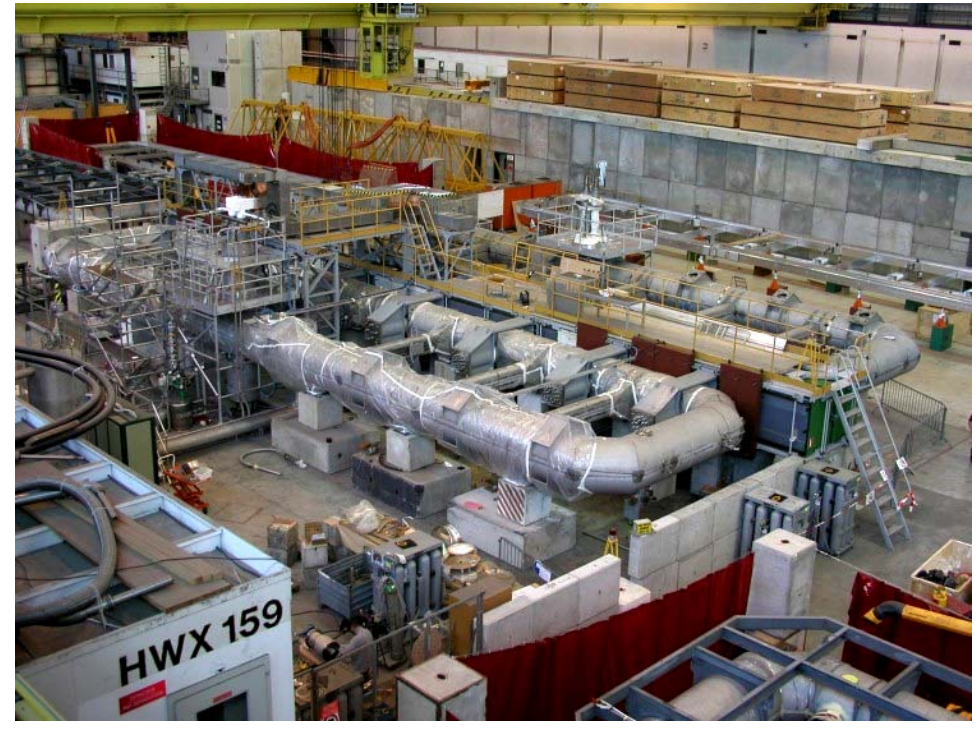

FIGURE 1. Top view of the experimental set-up. Two magnets are in place separated by an iron wall (magnetic mirror).The magnet in foreground is cooled at $4.5 \mathrm{~K}$, whilst the one in background is being prepared for the test.

\section{SET-UP OF THE CRYOGENIC FACILITY}

The experimental set-up (Fig. 1) has been described elsewhere [1] in connection to the test of the first prototype magnet at reduced scale [2]. Furthermore, the characteristics of the liquid helium (LHe) centrifugal pumps to be used for the coil tests were evaluated. Two centrifugal LHe pumps are immersed in a dewar that acts as a phase separator (PS) on the return line from the coil cooling circuits. The PS is connected to the refrigerator, which keeps the level of LHe constant. In Fig. 2 the characteristic curves of the two pumps are shown. The efficiency of the pumps [3] was also measured as being 0.35 at $80 \mathrm{~g} / \mathrm{s}$ and $30 \mathrm{kPa}$ (300 mbar).

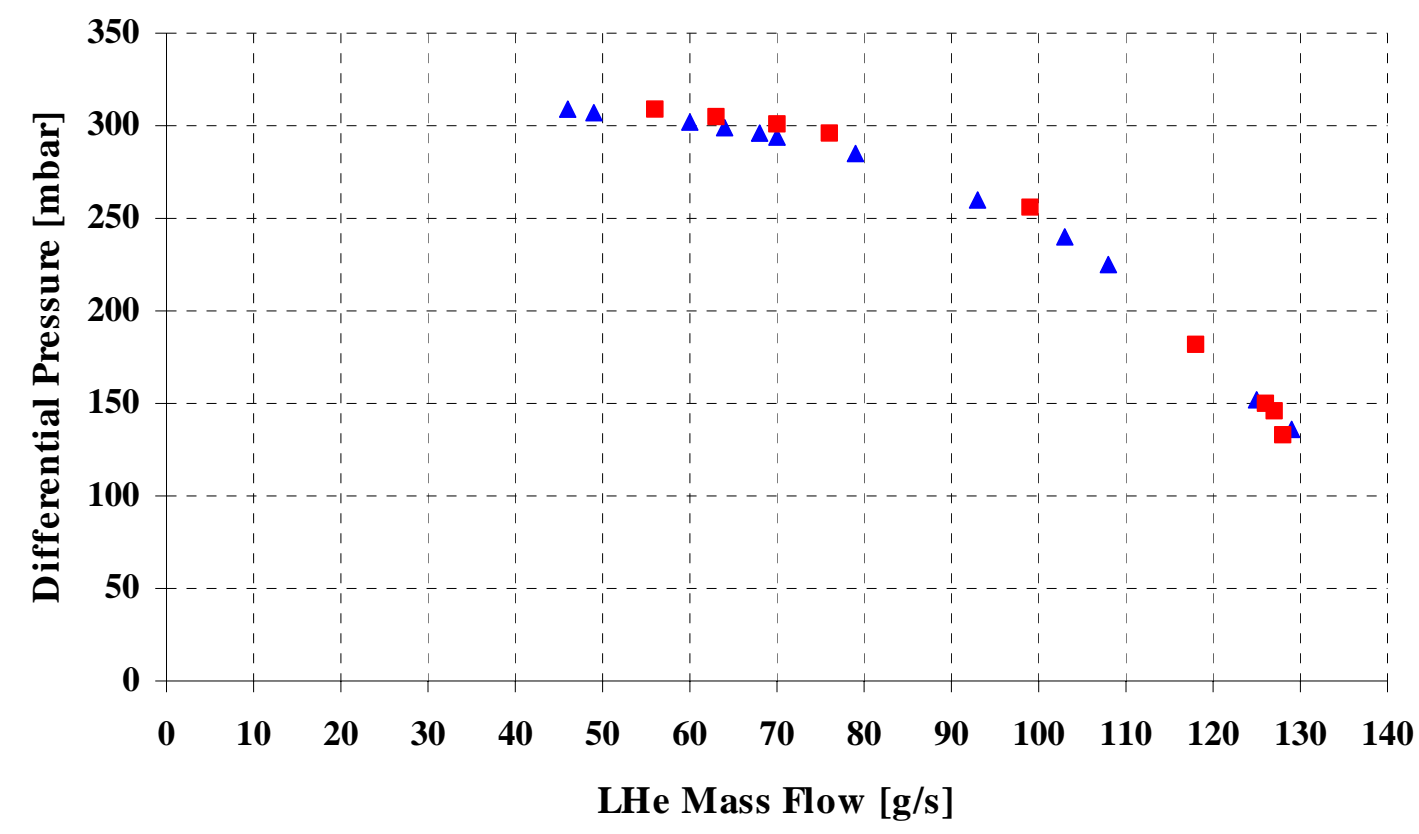

FIGURE 2. Characteristic curves of the two LHe centrifugal pumps used for the coil tests. The operating working point during the tests, as given by the impedance of the system with the pump running at $3950 \mathrm{rpm}$, was about 200 mbar and $110 \mathrm{~g} / \mathrm{s}$. 


\section{Typical Magnet Cool-down Curve}

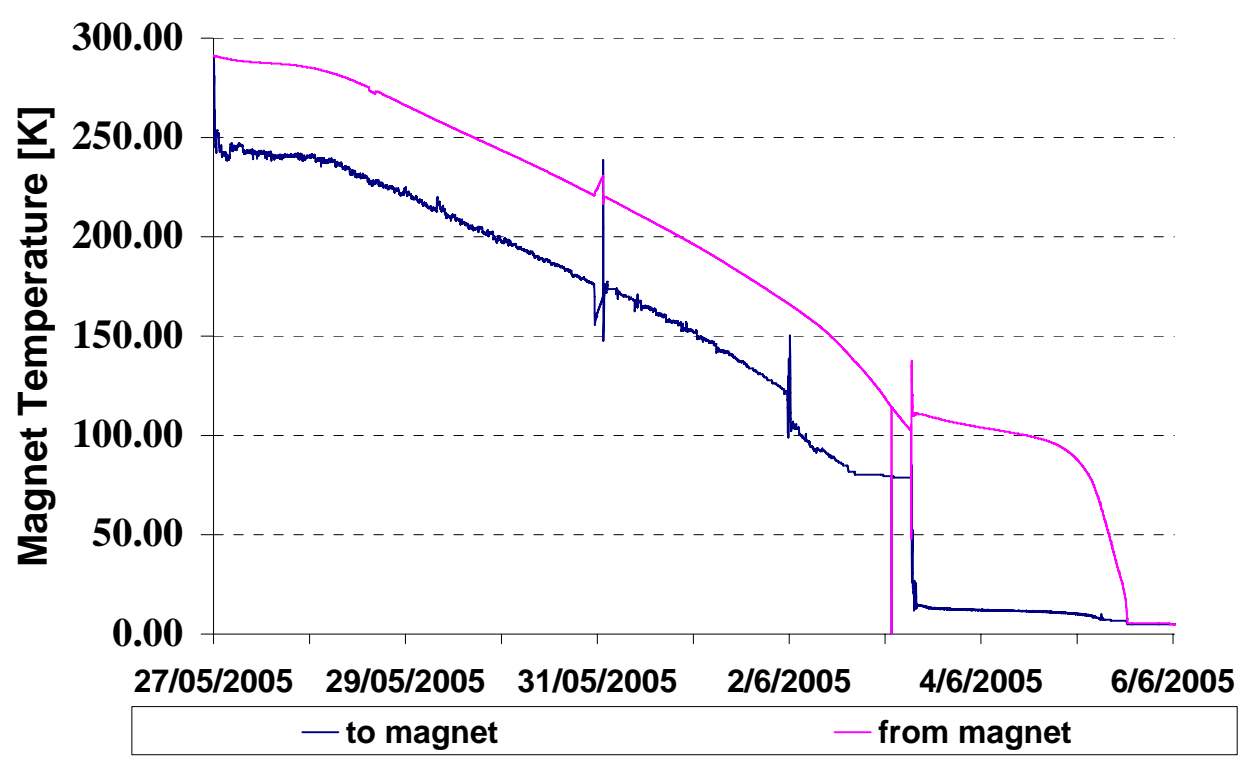

FIGURE 3. The curve shows a typical cooling down of a ATLAS BT coil. The line below refers to the supply temperature while the upper one refers to the return one. The transition, after seven days, from the pre-cooler to the refrigerator is visible, when supercritical helium is sent to the cold masses. Three additional days are needed to cool down to $4.5 \mathrm{~K}$.

The cryogenic system cools the ATLAS coils down from ambient temperature to about $80 \mathrm{~K}$ with a $10-\mathrm{kW}$ pre-cooler, which is essentially a counter-flow gaseous helium heat exchanger cooled by liquid nitrogen (LN2), and capable of maintaining a differential temperature of $40 \mathrm{~K}$ between the inlet and outlet. The gaseous helium, up to $50 \mathrm{~g} / \mathrm{s}$, is compressed by a vertical screw compressor to about $1.8 \mathrm{MPa}$ (18 bar) absolute. Cooling of the cold mass from $80 \mathrm{~K}$ to $4.5 \mathrm{~K}$ is achieved using a $1.2 \mathrm{~kW}$ at $4.5 \mathrm{~K}$ isothermal capacity refrigerator. The refrigerator additionally cools the thermal shields of the coil cryostat. A typical cool-down curve for a coil is shown in Fig. 3, where the transition from the precooler to the Main Refrigerator (MR) is indicated. At this point the MR supplies the cryogenic fluid directly to the cooling circuit of the coils, returning to the PS first and then to the MR. It takes about ten days to cool down a magnet to $4.5 \mathrm{~K}$ from ambient temperature, seven of which are with the pre-cooler to the $80 \mathrm{~K}$ level.

When LHe arrives in the phase separator and when an adequate level is present there, one of the two LHe pump is switched on to send LHe to the magnet: from this moment the refrigerator's duty is to keep the level constant in the PS.

It has to be noted that part of the LHe sent to the magnets is also diverted to the current leads (CL) and used to energize the magnet up to $22 \mathrm{kA}$. The helium exits the CL at ambient temperature and it is sent back to the suction side of the helium cycle compressor. The amount of LHe sent to the CL varies with the current passing through them. At nominal current $(20.5 \mathrm{kA})$ the helium flow was $1.7 \mathrm{~g} / \mathrm{s}$ on each of the CL, and $0.4 \mathrm{~g} / \mathrm{s}$ with zero current.

\section{DESCRIPTION OF THE TESTS}

The aim of the test, from the cryogenic point of view, was to measure the static and dynamic heat loads for each of the eight coils, and to compare the values to the calculated ones [4]. 


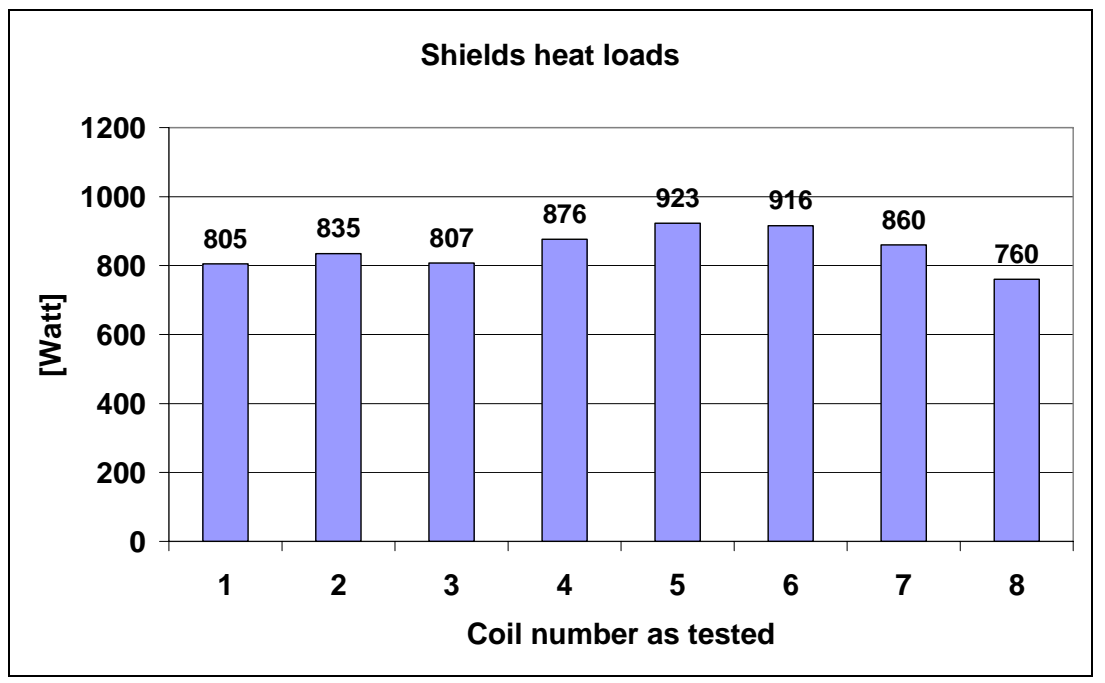

FIGURE 4. Summary of the heat loads of the eight thermal shields of the BT as measured at the average temperature of $70 \mathrm{~K}$. Typical estimated measurement errors are on the order of $10 \%$.

\section{Measurement of the Static Heat Loads}

The heat loads of the thermal shield circuits were obtained by measuring the gas helium mass flow and its differential temperature between the inlet and outlet. The mass flow was measured by means of a calibrated orifice. Its calibration was carried out by recording the pressure difference when changing the electrical power of a resistor immersed in the fluid. The average temperature of the shield during all measurements was $70 \mathrm{~K}$. The measured heat load results for the eight thermal shields are summarized in Fig. 4.

Considering the measurement errors, the results on the average are in good agreement with the evaluation of $740 \mathrm{~W}$ at $60 \mathrm{~K}$ done by CEA-SACLAY in [4].

The heat loads of the cold masses at $4.5 \mathrm{~K}$ were obtained by measuring the helium vapor mass flow returning to the refrigerator from the PS. The background heat load of the complete system, including the cryogenic transfer lines, the phase separator and the LHe centrifugal pumps, was measured separately for the two test stations.

The measuring method described differs from the one adopted for the magnet B0 [2], the reduced-scale prototype magnet. The measurement used here is quite delicate, the vapor mass flow being very sensitive to the slightest change in pressure and/or in the LHe level in the PS. Good stability of both parameters was normally achieved after about 40 minutes from the start, which turned out to be a limitation for the measurements of the dynamic heat loads, as discussed in the next section. A summary of the data collected is shown in Fig. 5. The results are in good agreement with the $79 \mathrm{~W}$ estimated in [4] with the only exception of coil no. 4. We cannot at present explain its very low heat load when compared to all the other ones.

\section{Dynamic Heat Load Measurements}

Eddy currents are induced in the aluminum casing and in the superconducting cable when the current changes in the coils. As a consequence, the cryogenic heat load at $4.5 \mathrm{~K}$ changes accordingly. The estimation of the dynamic loads at $4.5 \mathrm{~K}$ is very important for the correct operation of the refrigerating system of the ATLAS magnet complex. The electrical discharge of the magnet during a "slow dump," will be done at the final 


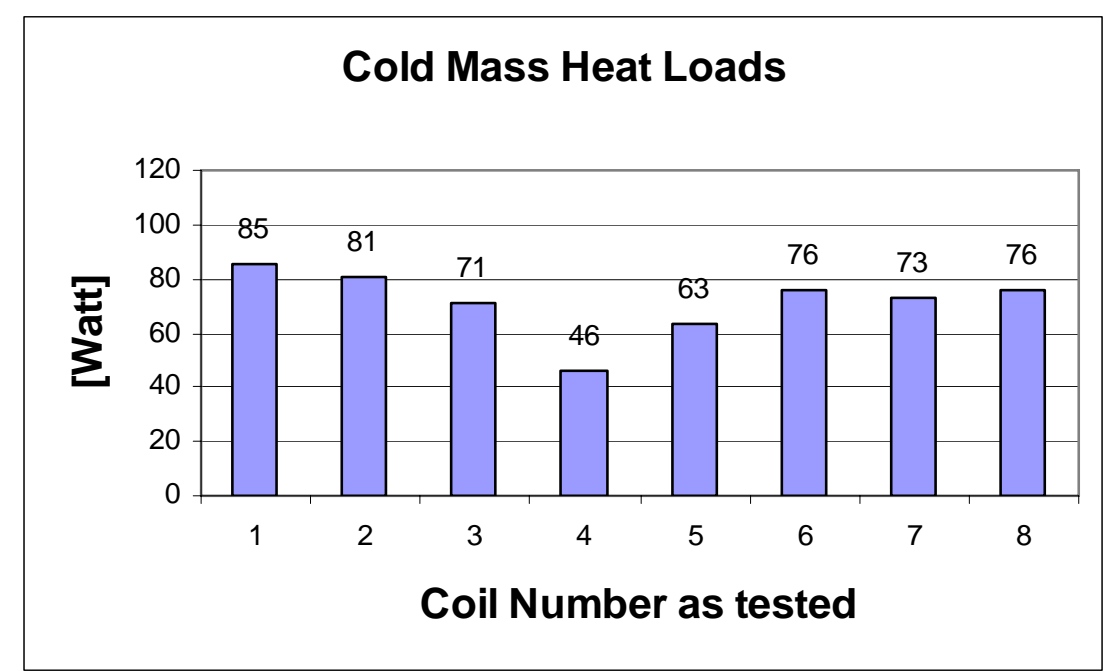

FIGURE 5. Summary of the static heat loads of the coil cold masses. The values are in good agreement (see text for details) with the ones evaluated in [4].

experiment site at a constant $\mathrm{dI} / \mathrm{dt}$. All the electromagnetic stored energy will be discharged into diodes at $4.2 \mathrm{~A} / \mathrm{s}$, i.e. in about 5000 seconds when charged at nominal current. It has to be noted that the slow dump is always preferred to the fast dump, since with the former the stored energy is dissipated in the diodes and maintaining the coil at $4.5 \mathrm{~K}$, while with the latter, the stored energy is dissipated in the cold mass in only three minutes, increasing its temperature up to $55 \mathrm{~K}$.

During the tests the equivalent of the dynamic load for a slow dump were simulated and measured, charging the magnet at a constant $\mathrm{dI} / \mathrm{dt}$, since the slow discharge in the test station is done into resistors and, as a consequence, $\mathrm{dI} / \mathrm{dt}$ is not constant. Data was collected for six of the eight coils: the values for the first and the seventh coil were not measured due to the instability of the cryogenic instrumentation. When compatible with the electromagnetic characteristics measurements program, charging at a constant $\mathrm{dI} / \mathrm{dt}[\mathrm{A} / \mathrm{s}]$ of 4, and 5, and 6 allowed the estimation of the dynamic heat load of the entire BT. For some of the coils only one point was measured. A summary of the values measured is shown in Fig. 6, where they are compared to the curve corresponding to the case when all the energy produced by eddy currents is induced in the aluminum casing [2].

The estimated error is around $15 \%$ : it can be seen that for the third coil tested, the deviance from the reference curve is higher than the estimated error. Nevertheless it has to be noted that for that particular coil, it was possible to measure only one value. The BT total inductance is $5.5 \mathrm{H}$, i.e. higher than eight times the single inductance $(\mathrm{L}=0.5667 \mathrm{H})$ of each race-track coil tested. Therefore the data collected results in a estimate of $350 \mathrm{~W}$ at $4.2 \mathrm{~A} / \mathrm{s}$ for the total dynamic load due to eddy currents for the eight coils that form the BT.

\section{RECOVERY AFTER A QUENCH}

The test program for each coil included inducing one or more quenches (fast dump) at different values of the maximum current. The average inductance being $\mathrm{L}=0.5667 \mathrm{H}$, the stored energy could reach $138 \mathrm{MJ}$ at the maximum tested value of $22 \mathrm{kA}$, so that the cold mass could eventually warm up to $55 \mathrm{~K}$ in a few minutes. A new automatic procedure was implemented to recover from a fast dump, permitting recovery and reestablishment of operation in less than a day (see Fig. 7).

After a fast dump the refrigerator is isolated from the PS and operated in a closed circuit in preparation for cool down of the coil to $4.5 \mathrm{~K}$. 


\section{Dynamic heat load}

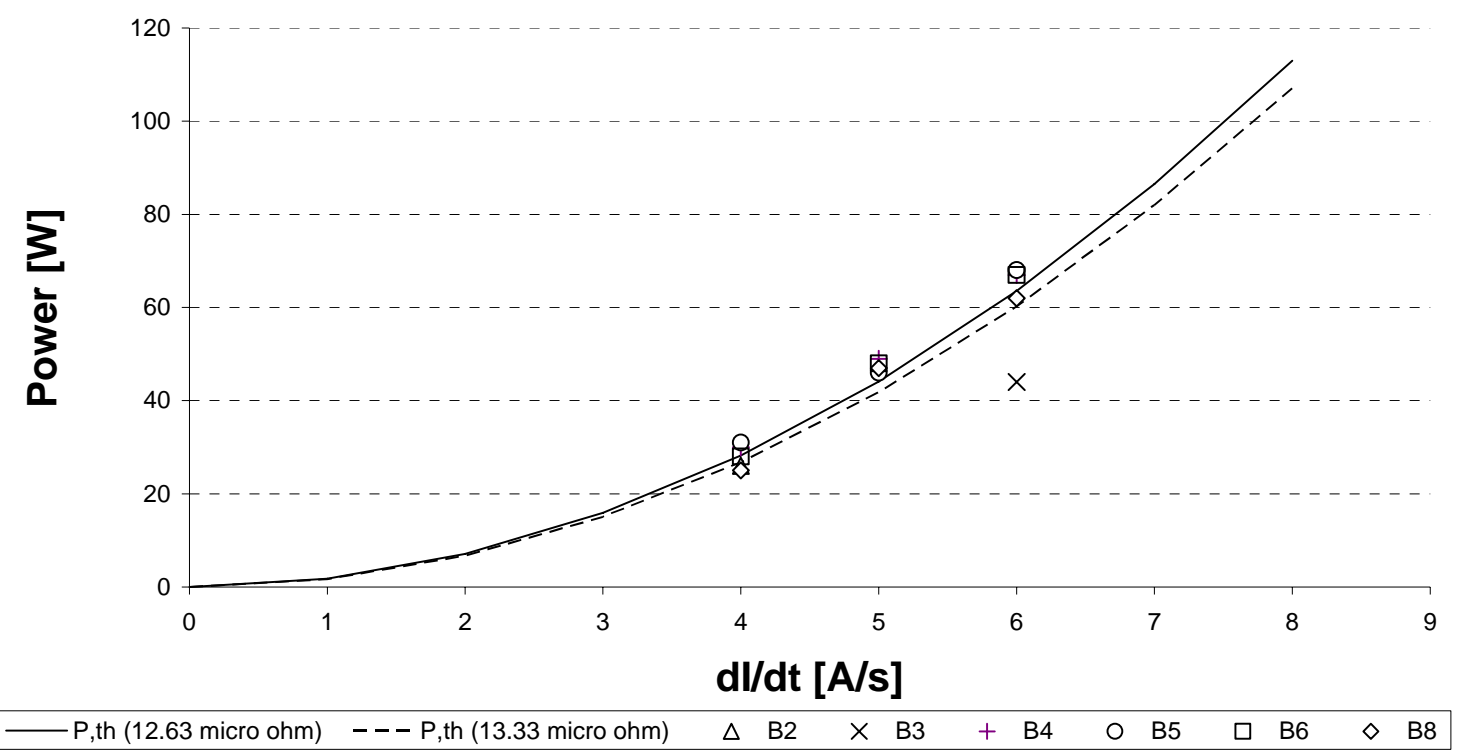

FIGURE 6. Summary of the dynamic heat load for the BT coils. The two curves correspond to the average inductance $\mathrm{L}=0.5667 \mathrm{H}$ and the casing total resistance of 13.3 and 12.65 micro-Ohm respectively as in [2].

The control-system functional logic was modified from the existing one [5] in order to recover and to be ready for another fast dump in about 18-24 hours. The recovery was carried out using helium at $0.26 \mathrm{MPa}(2.6 \mathrm{bar})$ and $4.5 \mathrm{~K}$ at the exit of the refrigerator, while at the entrance of the coil its temperature was about $5.5 \mathrm{~K}$. Fig. 7 shows a typical series of fast dumps and recoveries.

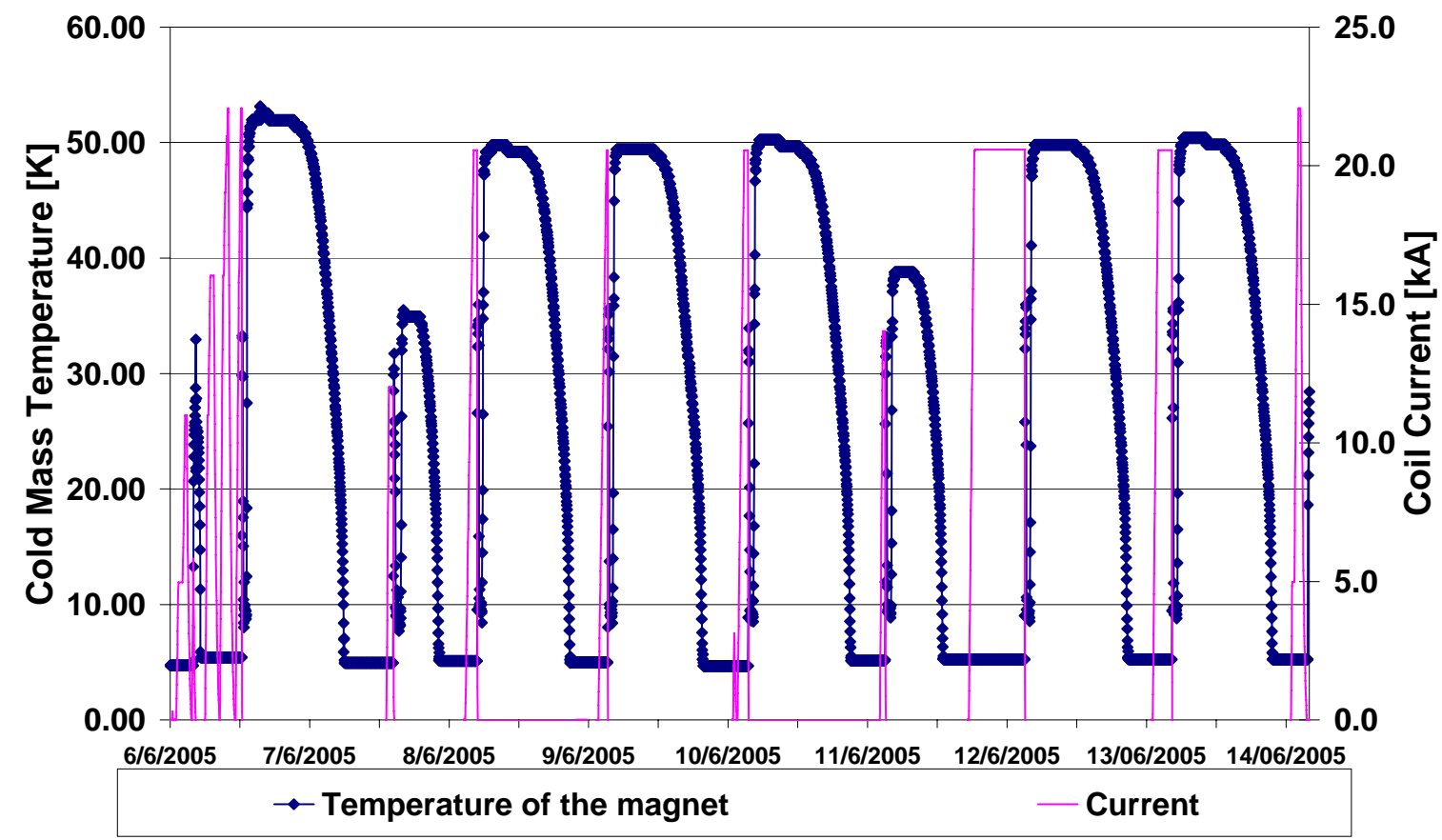

FIGURE 7. Shown are a series of recoveries from a fast dump. The first fast dump and recovery refers to the maximum current of $22 \mathrm{kA}$, five of them refer to the nominal current of $20.5 \mathrm{kA}$, and two to intermediate lower current values. 


\section{CONCLUSION}

In the paper the results of the test program for the eight coils forming the ATLAS Barrel Toroid, carried out prior to the installation in the experimental area underground have been described. A good overall agreement with the data collected from the prototype magnet and with the estimated values was found. A new operating procedure using supercritical helium permitting recovering in less than a day from a fast dump was also implemented.

\section{ACKNOWLEDGEMENTS}

The authors would like to express their thanks to J. L. Escourrou, E. Mattelon, F. Rodriguez, and all the operating team for their valuable support during the period of the tests and test preparation.

\section{REFERENCES}

1. F. Haug, et al., “The Cryogenic Test Facility for ATLAS Barrel Toroid Magnets,” MT-16, edited by B. Cornett and Y. Jensen, Ponte Vedra Beach, USA, September 1999, p.1514

2. R. Pengo, et al., "Heat Load Measurements on a Large Superconducting Magnet: an Application of a Void Fraction Meter,” MT-18, Ed. by T. Shintomi, Morioka City, Japan, October 2003, p.1704.

3. R. Pengo, et al. "Test Results of a $1.2 \mathrm{~kg} / \mathrm{s}$ Centrifugal Liquid Helium Pump for the ATLAS Superconducting Toroid Magnet System,” ICEC-19, edited by G. Gistau Baguer and P. Seyfert, Grenoble, France, July 2002, p.71.

4. C. Mayri, “Cryogenic Request Estimation,” CEA-Saclay Rapport Technique 5C-2900 T \# 6000007 PA, May 2003.

5. N. Delruelle, et al, "First Cryogenic Testing of a ATLAS superconducting Prototype Magnets,” MT-17, edited by S. Russenschuck, Geneva, September 2001, p.1336. 